\title{
Is the Critical Velocity Test a Good Tool For Aerobic Assessment of Children Swimmers?
}

\author{
Sousa $\mathrm{M}^{1, *}$, Vilas-Boas J.P $\mathrm{P}^{2}$ and Fernandes R.J ${ }^{2}$ \\ ${ }^{\prime}$ Centre of Research, Education, Innovation and Intervention in Sport - Faculty of Sport, University of Porto, Portugal \\ ${ }^{2}$ Centre of Research, Education, Innovation and Intervention in Sport - Faculty of Sport and Porto Biomechanics \\ Laboratory, University of Porto, Portugal
}

\begin{abstract}
Although swimmers are involved at very young ages in training and in competition, the differences in the physiological responses to exercise between them and adults are usually not respected. In fact, children swimmers are rarely involved in training control, leading to inadequate volume and prescription of training intensities. Our purpose was to verify if the critical velocity test is a good tool for aerobic assessment in children swimmers, by comparing it with the velocity corresponding to metabolic individual anaerobic threshold. Fourteen swimmers of $10.7 \pm 0.73$ years old voluntarily participated in the present study. Critical velocity was determined as the slope of the regression line between two competitive events (100 and $400 \mathrm{~m}$ freestyle), and the corresponding official times. In addition, each participant performed a $5 \times 200 \mathrm{~m}$ front crawl intermittent incremental protocol for individual anaerobic threshold assessment, with $30 \mathrm{~s}$ intervals and $0.05 \mathrm{~m} / \mathrm{s}$ increments between steps; the velocity at $4 \mathrm{mmol} / 1$ of blood lactate concentrations ([La $]$ ) was also determined by extrapolation of the [ $\left.\mathrm{La}^{-}\right] /$velocity curve. The mean values obtained were: $1.04 \pm 0.07,1.03 \pm 0.05$ and $1.08 \pm 0.06 \mathrm{~m} / \mathrm{s}$ for the critical velocity, velocity at anaerobic threshold and velocity at $4 \mathrm{mmol} / 1$ (respectively), being the first two parameters similar but lower than the velocity at $4 \mathrm{mmol} / \mathrm{l}$. These results confirm that the critical velocity test is of simple and practical implementation, using data from competition (or by implementing maximal tests in training context), allowing to assess in a non-invasive way the aerobic capacity of children swimmers.
\end{abstract}

Keywords: Aerobic assessment, anaerobic threshold, critical velocity, children swimmers.

\section{INTRODUCTION}

In the last decades, the participation of children in highlevel sports increased significantly, and the deeper understanding of their performance influencing factors and well-being during training and competition has gained great importance [1]. Particularly in swimming, children start their participation in training, and are engaged in competitive events, in a very young age ( $\sim 10$ years). Following the swimming specialized literature, at these ages, it is advised to be involved in 3-5 training units per week $(1-2 \mathrm{~h}$ per session), with a training volume of 2500 to $3000 \mathrm{~m}$ per session, and 7500 to $15000 \mathrm{~m}$ per week $[2,3]$. At this moment of a swimmer's career, practitioners are in the "basic training" phase, developing simultaneously the proper starting, swimming, and turning techniques, as well as their basic conditional skills (namely their aerobic capacity) to prepare for future intense high-volume training [4]. Although existing several differences between the physiological responses of children and adults to exercise [5], children swimmers are rarely involved in training control, and the investigation in these ages is very low comparing to that conducted with adult swimmers [6]; this seems to be due to financial limitations, but also to ethical

*Address correspondence to this author at Rua Dr. Plácido Costa, 91 4200450, Porto, Portugal; Tel: +351 225074763;

E-mail: smarisacsousa@hotmail.com issues [7]. The almost inexistence of swimmers evaluation and training control at young ages could lead to inadequate volume and prescription of training intensities, essentially based on coaches' previous experience [8], not considering the swimmers specific training responses [5, 9].

Knowing that swimming is an individual and cyclic sport, in which both conditional and biomechanical factors are determinant for the swimmer's performance [10], and that a higher percentage of swimming training is dedicated to aerobic performance improvement $[4,11]$, the training control and evaluation of the swimmer's aerobic performance is a fundamental tool for increasing the efficiency of training processes $[11,12]$, and to performance prediction [13]. To evaluate the swimmer's aerobic performance one of the most valid indicators is the individual anaerobic threshold, as it gives important information regarding the level of development of the swimmer's aerobic capacity [4, 6, 14-16], being very useful to establish the aerobic training intensities in swimming. However, despite the existence of several tests to assess the velocity corresponding to the anaerobic threshold, some imply the use of invasive techniques based in expensive procedures of blood collection for lactate concentration analysis, and, frequently, are also very time consumable [10].

Trying to overcome the above referred constraints, Wakayoshi et al. [17] developed and adapted to swimming the concept of critical power introduced by Monod and 


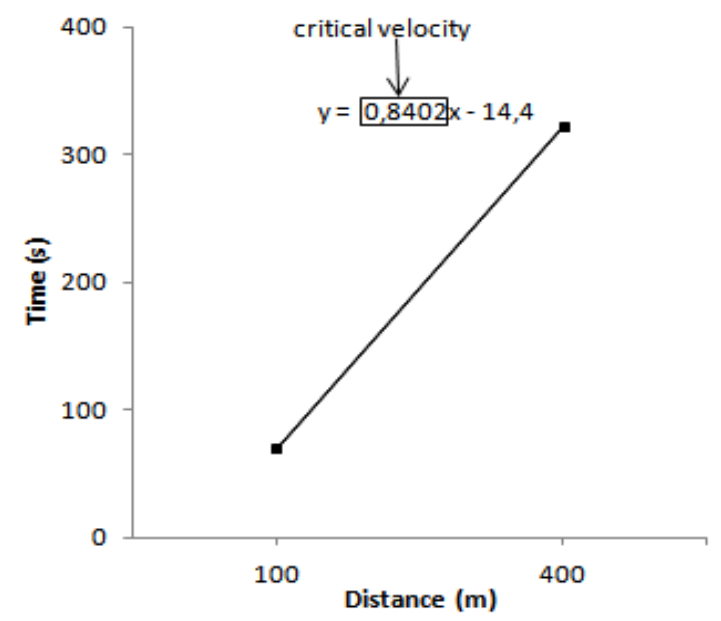

Fig. (1). Example of the critical velocity determination using two competitive events.

Scherrer [18] for the total work done by one muscle or one synergistic muscle group, presenting the critical velocity test. According to those authors, critical velocity corresponds to the maximal swimming velocity that can be maintained for a long period of time without exhaustion, being a parameter well related with the individual anaerobic threshold $[19,20]$. This parameter has been used as an indicator of swimming aerobic capacity in adult swimmers [17, 19], being also appropriate for aerobic training in adolescent swimmers [21, 22]. The critical velocity test is a simple, non-invasive and non-expensive methodology, which can be easily implemented in the training context, being obtained through the relationship between test or competitive distances and the time necessary to perform them at maximum intensity [17]. The purpose of the present study was to verify if the critical velocity test is a good tool for aerobic assessment in children swimmers, by comparing its outputs with the velocity corresponding to metabolic individual anaerobic threshold; comparisons with velocity corresponding to $4 \mathrm{mmol} / 1$ of blood lactate concentration ([ $\mathrm{La}^{-}$ ]), a mean value considered as the "gold standard" for aerobic capacity assessment, were also done.

\section{METHODS}

Fourteen swimmers $(10.7 \pm 0.73$ years old; body mass $40.6 \pm 7.04 \mathrm{~kg}$; height $148.9 \pm 7.15 \mathrm{~cm}$; arm span $149.1 \pm$ $9.77 \mathrm{~cm}$ and swimming experience $5.63 \pm 1.69$ years) voluntarily participated in the present study. All children trained 4 times per week, covering 12000-14000 m per week, mainly at aerobic regimens. Their performance at 100 and $400 \mathrm{~m}$ freestyle, using the front crawl technique, were $80.40 \pm 4.47$ and $371.61 \pm 21.05 \mathrm{~s}$, respectively. The criterion for children's participation was a performance of $\leq 180 \mathrm{~s}$ at the $200 \mathrm{~m}$ front crawl event. The local ethics committee approved the procedures, and all the swimmers' parents signed a consent form in which the protocol was explained.

The critical velocity was determined as the slope of the regression line between two distances obtained in competition (100 and $400 \mathrm{~m}$ freestyle, performed in front crawl), and the correspondent official time. In Fig. (1) it is possible to observe, for one swimmer, the critical velocity result represented as the slope of the regression line ("a" value, expressed in $\mathrm{m} / \mathrm{s}$ ) from the distance $(\mathrm{y})$ in function of the time (x) relationship; the " $\mathrm{b}$ " value is the y-interception value, according to the equation $\mathrm{y}=\mathrm{ax}+\mathrm{b}$.

One week before the competition, each participant performed a $5 \times 200 \mathrm{~m}$ front crawl intermittent incremental protocol for individual anaerobic threshold assessment, with $30 \mathrm{~s}$ intervals and $0.05 \mathrm{~m} / \mathrm{s}$ increments between steps, as described in deeper detail by Fernandes et al. [6]. A standardized warm-up of $600 \mathrm{~m}$, consisting primarily of aerobic swimming of low to moderate intensity, was conducted before the test protocol. All tests were conducted in a $25 \mathrm{~m}$ indoor swimming pool, $1.90 \mathrm{~m}$ deep, with a water temperature of $27^{\circ} \mathrm{C}$.

Descriptive statistics (means and standard deviations) were obtained for all variables (all data were checked for normality of distribution using the Shapiro-Wilk test). Pearson's correlation coefficient, pair wise $t$ test, and Bland-Altman test were also used. A significance level of $5 \%$ was accepted.

\section{RESULTS}

It is possible to observe in Table $\mathbf{1}$ the individual, mean and standard deviation values of the critical velocity, the velocity corresponding to the individual anaerobic threshold, and the velocity corresponding to $4 \mathrm{mmol} / 1$ of $\left[\mathrm{La}^{-}\right]$, by gender and for the total sample.

For the total sample and each gender subgroup the critical velocity values are similar to the velocity at individual anaerobic threshold. However, the critical velocity is significantly lower than velocity at $4 \mathrm{mmol} / 1$ of [ $\left.\mathrm{La}^{-}\right]$in the total sample and in the female group (in the male group a tendency for lower values is also observable); these differences correspond to a $\sim 5 \mathrm{~s}$ gap in a $100 \mathrm{~m}$ front crawl effort when the total sample is considered. Nevertheless, all parameters were positively correlated (Table $\mathbf{2}$ ).

The Fig. (2) shows the Bland-Altman diagrams comparing critical velocity and velocity at individual anaerobic threshold (A panel), critical velocity and velocity at $4 \mathrm{mmol} / 1$ of [ $\left.\mathrm{La}^{-}\right]$(B panel), and velocity at individual anaerobic threshold and velocity at $4 \mathrm{mmol} / 1$ of [ $\left.\mathrm{La}^{-}\right]$(C panel).

The repeatability coefficient [and $95 \%$ agreement limits], as described by Bland and Altman [23] were: (i) $0.007 \mathrm{~m} / \mathrm{s}$ [0.015 to 0.029 ] for critical velocity - velocity at individual anaerobic threshold; (ii) -0.049 [-0.075 to -0.023$]$ for critical velocity - velocity at $4 \mathrm{mmol} / 1$ of [ $\left.\mathrm{La}^{-}\right]$; and (iii) $-0.056 \mathrm{~m} / \mathrm{s}[-$ 0.072 to 0.041$]$ for velocity at individual anaerobic threshold - velocity at $4 \mathrm{mmol} / 1$ of [ $\left.\mathrm{La}^{-}\right]$, respectively.

\section{DISCUSSION}

It is accepted for some time that the improvement of swimmer's performance can no longer be obtained only by increasing the training volume and using nonspecific methodologies, but by carefully designing the training process and systematically monitoring it $[4,8]$. Given that a high percentage of the training volume is dedicated to the improvement of the swimmer's aerobic capacity [11], particularly at young ages [3-5], coaches need objective data that allow them to, complementarily to the improvement of the swimmer's technical skills, better prescribe the adequate intensities to develop the aerobic performance [10]. This is particularly true for infant swim training programs once it is 
Table 1. Individual Mean and SD Values for Critical Velocity (CV), Velocity at Individual Anaerobic Threshold (IndAnTv) and Velocity at 4mmol/l of [ $\left.\mathrm{La}^{\top}\right]$ (v4), in Male and Female Groups, and in the Total Sample

\begin{tabular}{|c|c|c|c|}
\hline & CV $(\mathbf{m} / \mathbf{s})$ & IndAnTv (m/s) & v4 (m/s) \\
\hline \hline 1 & 1.19 & 1.12 & 1.15 \\
\hline 2 & 1.15 & 1.09 & 1.17 \\
\hline 3 & 1.08 & 1.08 & 1.19 \\
\hline 4 & 1.04 & 1.01 & 1.12 \\
\hline 5 & 1.06 & 1.06 & 1.03 \\
\hline 6 & 1.01 & 1.00 & 1.07 \\
\hline 7 & 0.98 & 1.03 & $1.10 \pm 0.06^{\mathrm{b}}$ \\
\hline Mean SD & $1.07 \pm 0.07$ & $1.05 \pm 0.05^{\mathrm{a}}$ & 1.11 \\
\hline 9 & 1.06 & 1.08 & 1.06 \\
\hline 10 & 1.06 & 1.02 & 1.08 \\
\hline 12 & 1.04 & 1.05 & 1.03 \\
\hline 13 & 0.97 & 0.97 & 1.06 \\
\hline 14 & 0.96 & 1.01 & 1.07 \\
\hline 15 & 0.95 & 0.98 & 1.02 \\
\hline Mean \pm SD & 0.98 & 0.93 & $1.06 \pm 0.03^{\mathrm{b}, \mathrm{c}}$ \\
\hline Mean Total & $1.00 \pm 0.05^{\mathrm{a}}$ & $1.00 \pm 0.05^{\mathrm{a}}$ & $1.08 \pm 0.06^{\mathrm{b}, \mathrm{c}}$ \\
\hline
\end{tabular}

Significant differences between variables are represented by ${ }^{\mathrm{a}} \mathrm{v} 4,{ }^{\mathrm{b}} \mathrm{IndAnTv},{ }^{\mathrm{c}} \mathrm{CV}$ for $\mathrm{p} \leq 0.05$.

Table 2. Correlation Matrix Obtained Between the Critical Velocity (CV), Velocity at Individual Anaerobic Threshold (IndAnTv) and Velocity at $4 \mathrm{mmol} / \mathrm{l}$ of $\left[\mathrm{La}^{-}\right]$(v4). All Parameters were Significantly Correlated $(\mathrm{p}<0.01)$

\begin{tabular}{|c|c|c|c|}
\hline & $\mathbf{C V}$ & IndAnTv & v4 \\
\hline \hline $\mathrm{CV}$ & 1 & & 1 \\
\hline IndAnT & $.84^{* *}$ & $.88^{* *}$ & 1 \\
\hline $\mathrm{v} 4$ & $.77^{* *}$ & & \\
\hline
\end{tabular}

Significant correlations are represented by $* *(p \leq 0.01)$
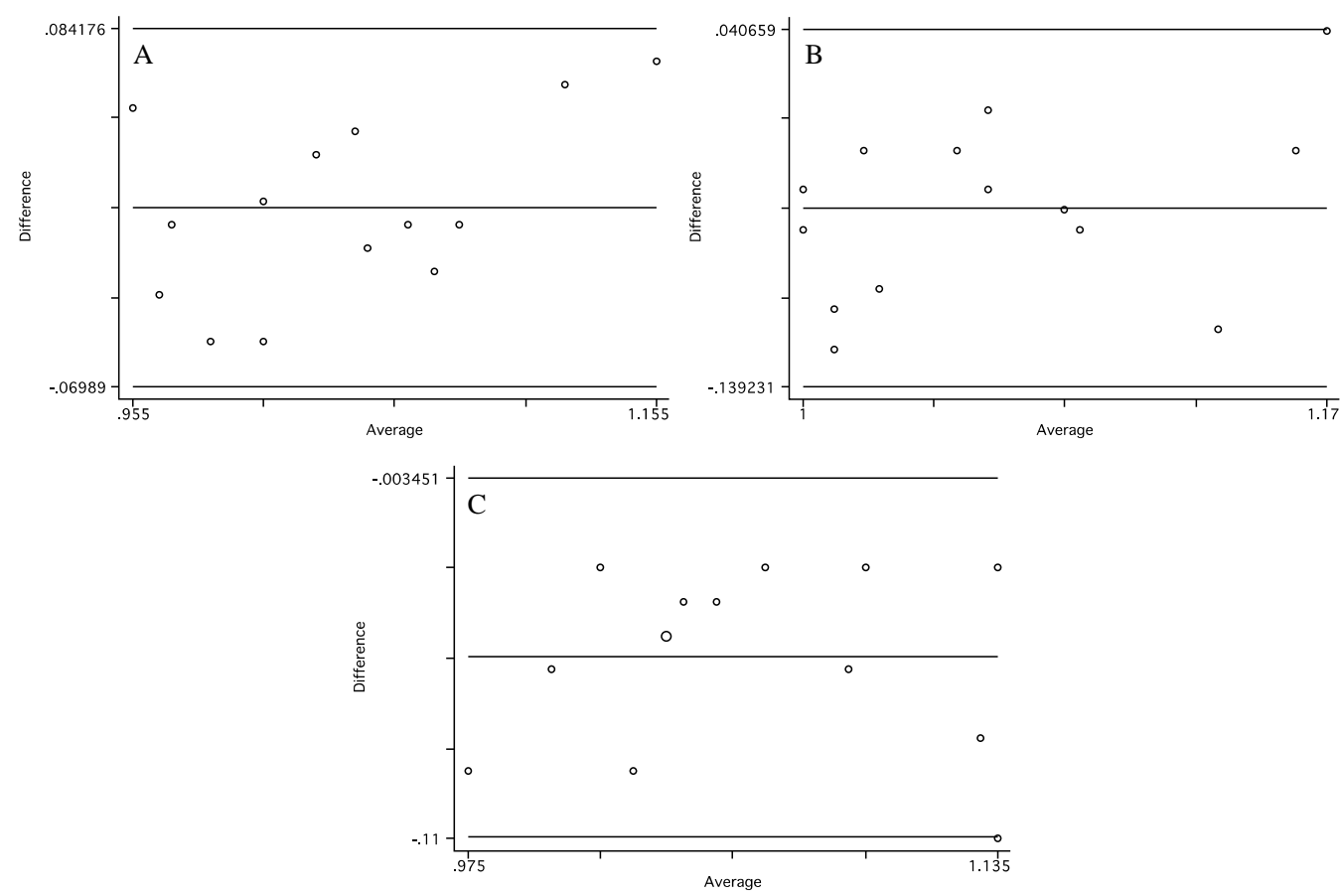

Fig. (2). Bland-Altman diagram comparing critical velocity and velocity at individual anaerobic threshold (panel A), critical velocity and velocity at $4 \mathrm{mmol} / 1$ of [ $\left.\mathrm{La}^{-}\right]$(panel B), and velocity at individual anaerobic threshold and velocity at $4 \mathrm{mmol} / 1 \mathrm{of}\left[\mathrm{La}^{-}\right]$(panel C). 
common to see children in early elementary school engaged in several hours of practice each week throughout the year. Therefore, more objective and specific training programs are required to develop the training process quality (aiming to improve performance), which is possible through the training control process [10]. However, physiologic assessment in children should not be expensive, invasive, complex and time consuming comparing to the battery of tests carried-out in adult swimmers [7, 24].

Although the "gold standard" for the evaluation of the aerobic capacity, by assessing the balance between lactate production and its removal, is the Maximal Lactate Steady State test [33], its implementation is rather difficult once: (i) it is required several samples of blood (at least four in each bout, repeating it each trial); (ii) swimmers need to perform, in general, three $30 \mathrm{~min}$ trials at different velocities, which demands high levels of motivation. In addition, to avoid the blood collection required for the assessment of the metabolic anaerobic threshold (an invasive and expensive method), age-group coaches usually implement simple non-invasive distance tests, particularly the $30 \mathrm{~min}$ continuous swim test T30 [4] - and the $2000 \mathrm{~m}$ [25] swim test. However, the referred tests contain significant limitations preventing coaches to use them mainly when testing high number of children swimmers: (i) as the tests are performed in training conditions (with swimmers performing very close from each other), the final result of the swimmers that followed the line leader could be adulterated due to the drafting phenomenon and (ii) the continuous long distance tests are boring, not motivating the children to give an effective effort during the testing.

The critical velocity methodology seems to be a good alternative for aerobic monitoring in young ages, once it is simple, non-invasive and non-expensive, being easily implemented using competition distances, as previous conducted by our group [21]. The critical velocity test could also be implemented in a training context by performing maximal tests [10], once two bouts are perfectly conducted in a training unit carrying out the shorter distance after the warm up, and the longer distance after a regenerative training series of, at least, 45 min duration. As Wright and Smith [13] advised to do not suppress a long test distance when carrying out a critical velocity test, once it may lead to an overestimation of the final result, and as Dekerle et al. [26] proposed its duration to be within 2 to $30 \mathrm{~min}$, the use of 100 and $400 \mathrm{~m}$ distances (with a mean duration of $79 \pm 4 \mathrm{~s}$ and $373 \pm 22 \mathrm{~s}$, respectively) seem to be well justified.

The critical velocity mean value obtained in the current study was very similar, and strongly related, with the velocity at the individual anaerobic threshold, corroborating the studies of Wakayoshi et al. [17] and Wakayoshi et al. [27] conducted in adult swimmers. This fact evidences that the critical velocity test allows assessing the maximal velocity of a swimmer in a physiological aerobic balanced regimen $[20,28]$, and that it is useful for prescribing specific training intensities to the aerobic capacity development [21, 22] in these children swimmers. Based on the critical velocity values obtained in this study, and as it is accepted that for developing aerobic capacity efforts lasting $\sim 30 \mathrm{~min}$ are required, it is purposed a training series of $2 \times(7 \times 100$ $\mathrm{m}$, each $2 \mathrm{~min}$ ) $\mathrm{m}$ front crawl, with $400 \mathrm{~m}$ recovery between sets. As a group, these swimmers should complete every 100 $\mathrm{m}$ repetition in $95 \mathrm{~s}$, resting $25 \mathrm{~s}$, to effectively develop aerobic capacity. As expected, the obtained critical values are lower than those described for older swimmers [13, 17, 21, 22, 29, 30].

The significantly higher values of the velocity at 4 $\mathrm{mmol} / \mathrm{l}$ of $\left[\mathrm{La}^{-}\right]$compared to critical velocity values confirm that these parameters are not coincident and interchangeable, as noticed before in young and post pubertal [22] and juvenile swimmers [21]; in fact, the significant difference obtained between them - close to $5 \mathrm{~s}$ for a $100 \mathrm{~m}$ effort seems to show that, in these swimmers, the velocity at 4 $\mathrm{mmol} / \mathrm{l}$ of $\left[\mathrm{La}^{-}\right]$is not representative of the velocity at the individual anaerobic threshold. As previous described [15, $30-33]$, the velocity at $4 \mathrm{mmol} / 1$ of $\left[\mathrm{La}^{-}\right]$overestimates the velocity corresponding to individual anaerobic threshold, and limits the use of the reference velocity at $4 \mathrm{mmol} / \mathrm{l}$ of [ $\left.\mathrm{La}^{-}\right]$value to assess the proper intensities to develop swimming aerobic capacity. The velocity at $4 \mathrm{mmol} / 1$ of [ $\mathrm{La}^{-}$ ] was closer to the velocity obtained in the $400 \mathrm{~m}$ distance, reinforcing that this parameter is a non-specific and inappropriate intensity to be used in the aerobic training.

\section{CONCLUSION}

It was confirmed that the critical velocity test is a good tool for aerobic assessment in children swimmers once its outputs are similar and well related with the velocity corresponding to metabolic individual anaerobic threshold. The critical velocity test is of simple and practical implementation, allowing obtaining objective data in real time to use for evaluating swimmers and to control and prescribe aerobic conditioning.

\section{CONFLICT OF INTEREST}

The authors confirm that this article content has no conflicts of interest.

\section{ACKNOWLEDGEMENT}

Declared none.

\section{REFERENCES}

[1] Baxter-Jones AG, Mundt C. The young athlete. In: Armstrong N, Ed. Paediatric exercise physiology - Advances in sport and exercise science. Philadelphia: Churchill Livingston Elsevier 2007; pp. 299-324.

[2] Wilke K, Madsen O. El entrenamiento del nadador juvenil. Buenos Aires: Editorial Stadium 1990.

[3] Maglischo E. Swimming Even Faster. Califórnia: Mayfield Publishing. Company 1993.

[4] Olbrecht J. The science of winning. Planning, periodizing and optimizing swim training. Luton, England: Swimshop 2000.

[5] Bar-Or O. Anaerobic performance. In: David Docherty, Ed Measurement in pediatric exercise science. Windsor, Canada: Human Kinetics 1996.

[6] Fernandes R, Sousa M, Pinheiro A, Vilar S, Colaço P, Vilas-Boas JP. Assessment of individual anaerobic threshold and stroking parameters in 10-11 years-old swimmers. Eur J Sport Sci 2010; 10(5): 311-7.

[7] Garrido N, Marinho DA, Reis VM, et al. Does combined dry land strength and aerobic training inhibit performance of young competitive swimmers? J Sports Sci Med 2010; 9: 300-10.

[8] Costill DL, Maglischo EW, Richardson A. Swimming. London: Blackwell Scientific Publications 1992.

[9] Armstrong N, Tomkinson RG, Ekelund U. Aerobic fitness and its relationship to sport, exercise training and habitual physical activity during youth. Br J Sports Med 2011; 45: 849-58. 
[10] Fernandes R. Aerobic evaluation of young swimmers using the critical velocity test brief report. J Phys Educ Sport 2011; 11(2): 215-20.

[11] Maglischo EW. Swimming fastest. Champaign, Illinois: Human Kinetics 2003.

[12] Mujika I, Chatard JC, Busso T, Geyssant A, Barale F, Lacoste L. Effects of training on performance in competitive swimming. Can J Appl Phys 1995; 20(4): 395-406.

[13] Wright B, Smith DJ. A protocol for the determination of critical speed as an index of swimming endurance performance. In: Miyashita M, Mutoh Y, Richardson AB, Eds. Medicine and Science in Aquatic Sports. Basel: Karger 1994; vol. 39: pp. 55-9.

[14] Heck H, Mader A, Hess G, Mucke S, Muller R, Hollmann W. Justification of the $4 \mathrm{mmol} / \mathrm{l}$ lactate threshold. Int J Sports Med 1985; 6: 117-30.

[15] Simon G. The role of lactate testing in swimming: 1997 Proceedings of the XII FINA World Congress on Sports Medicine. Gotegorg: Sweden 1997; pp.259-62.

[16] Pyne DB, Lee H, Swanwick K. Monitoring the lactate threshold in world-ranked swimmers. Med Sci Sports Exerc 2001; 33(2): 291-7.

[17] Wakayoshi K, Ikuta K, Yoshida $\mathrm{T}$, et al. Determination and validity of critical velocity as an index of swimming performance in the competitive swimmer. Eur J Appl Phys 1992a; 64: 153-7.

[18] Monod H, Scherrer J. The work capacity of synergic muscular group. Ergonomics 1965; 8: 329-38.

[19] Dekerle J, Sidney M, Hespel JM, Pelayo P. Validity and reliability of critical speed, critical stroke rate, and anaerobic capacity in relation to front crawl swimming performances. Int J Sports Med 2002; 23(2): 93-8.

[20] Wakayoshi K, Yoshida T, Udo M, et al. Does critical swimming velocity represent exercise intensity at maximal lactate steady state? Eur J Sports Med 1993; 6: 74-7.

[21] Fernandes R, Vilas-Boas JP. Critical velocity as a criterion for estimating aerobic training pace in juvenile swimmers. In: Keskinen K, Komi P, Hollander P, Eds. Proceedings of the VIII International Symposium of Biomechanics and Medicine in Swimming. University of Jyvaskyla, Finland: Gummers Printing House 1999; pp. 233-8.

[22] Toubekis AG, Tsami AP, Tokmakidis SP. Critical velocity and lactate threshold in young swimmers. Int J Sports Med 2006; 27 : 117-23.
[23] Bland JM, Altman DG. Statistical methods for assessing agreement between two methods of clinical measurement. Lancet 1986; 1: 307-10.

[24] Fernandes R, Aleixo I, Soares S, Vilas-Boas JP. Anaerobic critical velocity: a new tool for young swimmers training advice. In: Beaulieu NP, Ed. Physical Activity and Children: New Research. NY: Nova Science Publishers Inc 2008; 211-23.

[25] Touretski G. Japan official swimming coach clinic. Tokyo: Japan Amateur Swimming Federation 1993.

[26] Dekerle J, Pelayo P, Sydney M, Marais G. Determination of critical speed in relation to front crawl swimming performances. In: Paris P, Pigozzi F, Prinzi G, Eds, Proceedings of the $4^{\text {th }}$ Annual Congress of the European Colleague of Sport Science. Rome, Italy: University Institute of Motor Sciences 1999; p.129.

[27] Wakayoshi K, Yoshida,T, Udo M, et al. A simple method for determining critical speed as swimming fatigue threshold in competitive swimming. Int J Sports Med 1992; 13: 367-71.

[28] Barden JM, Kell RT. Relationships between stroke parameters and critical swimming speed in a sprint interval training set. J Sports Sci 2009: 27(3): 227-35.

[29] Ikuta Y, Wakayoshi K, Nomura T. Determination and validity of critical swimming force as performance index in tethered swimming. In: Troup JP, Hollander AP, Strasse D, Trappe SW, Cappaert JM, Trappe TA, Eds. Biomechanics and medicine in swimming VII; London: E \& FN SPON; 1999; pp. 146-51.

[30] Espalda M, Alves F. Critical Velocity and the Velocity at Maximal Lactate Steady State in Swimming. In: Kjendlie PL, Stallman RK, Cabri J, Eds. Proceedings of the XI Biomechanics and Medicine in Swimming. Oslo: Norway 2010; pp. 194-6.

[31] Kelly M, Gibney G, Mullins J, Ward T, Donne B, O'Brien M. A study of blood lactate profiles across different swim strokes. In: MacLaren M, Reilly T, Lees A, Eds. Biomechanics and medicine in swimming: Swimming science VI. London: England 1992; pp. 227-33.

[32] Stegmann H, Kindermann W, Schnabel A. Lactate kinetics and Individual Anaerobic Threshold. Int J Sports Med 1981; 2: 160-5.

[33] Ribeiro L, Balikian P, Malachias P, Baldissera V. Stage length, spline function and lactate minimum swimming speed. J Sports Med Phys Fitness 2003; 43: 312-8. 